\title{
WHAT THE BEES KNOW AND WHAT THEY DO NOT KNOW
}

\author{
L. FEJES TÓTH
}

In the first part of this paper we construct a more economical honeycomb than that of the hive bees for any parameters involved in the problem. The second part gives a survey of some further solved and unsolved isoperimetric problems concerning cell-aggregates.

I. Honeycombs. The honeycomb of the bees is a loose tissue of wax forming a plane layer. The first things on it which catch one's eye are the regular hexagonal patterns on both sides. The hexagons are the openings of prismatic vessels, called bee-cells. Kepler described the shape of the bee-cells more fully. It turned out that the bottom of a cell consists of three equal rhombi as shown in Figure 1b. Thus the two kinds of cells having their openings in opposite directions are separated by a zigzagged surface and not by a plane, as one would expect at first.

Why do the bees build such a strange conformation?

According to a widely spread hypothesis, going back to Pappus, the bees aim at economy: If, by some reason, the volume of a cell and the width of the whole layer are given, they try to use the minimum amount of wax per cell. Although among the various effects which interact in producing the honeycomb the utilitarian human motive attributed to the bees seems to play the least part, the above hypothesis was the source of highly interesting investigations. Thus we accept this hypothesis and try to point out what the bees do well and what they do not do well from the point of view of making the surface-area of their cells small.

To give the problem a precise formulation we define a honeycomb as a set of congruent convex polyhedra, called cells, filling the space between two parallel planes without overlapping and without interstices in such a way that

(1) Each cell has a face, called base (or opening) on one of the two planes but does not have faces on both planes.

(2) In the congruence of two cells their bases correspond to each other.

The distance between the parallel planes is the width of the honeycomb.

An address delivered before the Chicago meeting of the Society on April 24, 1964 by invitation of the Committee to Select Hour Speakers for Western Sectional Meetings; received by the editors February 14, 1964. 
Let $w$ be the width of a honeycomb, $v$ the volume of one of its cells and $a$ the area of the base of a cell. Consider a large cylindrical section of the honeycomb, the area of a base-circle of which equals $A$. Since the two base-circles contain approximately $2 A / a$ openings, the cylinder contains approximately the same number of cells. Thus the volume $A w$ of the cylinder equals approximately $2 A v / a$. This argument, which can easily be made precise, shows that $w a=2 v$. The area $a$ of the openings being uniquely determined by $v$ and $w$, it is all the same whether, in the following problem, we consider the cells as open vessels, the surface of which consists only of the internal faces, or as closed polyhedra.

We now formulate the

First isoperimetric problem for honeycombs. Among the polyhedra of volume $v$ generating a honeycomb of width $w$ find that one of least surface-area.

Though the solution of this problem is not known as yet, the following considerations will give us useful information.

In the honeycomb of the bees $w$ is large in proportion to $\sqrt[3]{v}$. In such a case it is reasonable to erect right prisms of height $w / 2$ on the bases. Something may be gained by a suitable formation of the bottoms, but the solution will depend, above all, on the shape of the base. In this respect the bees make a good choice, because of all convex plane-fillers of given area the regular hexagon has the least perimeter.

Choosing regular hexagonal bases the question of the most economical bottom-figure arises. In the rhombic bottom-figure we have one degree of freedom: we can turn the planes of the rhombi around their "horizontal" diagonals without changing $v$ and $w$. In which position will the surface-area be minimal? The history of the solution of this famous problem, raised by Réaumur, and the deep impression it made have been described in some books (see e.g. [1]). It turned out that, in close accordance with the shape of the bee-cells, the rhombi must include an angle equal to $120^{\circ}$. Thus if for some reason the bees stick to a rhombic bottom-figure, they do excellent work.

Before proceeding with the case of "deep" cells we make a remark which will give us an orientation in the general case. Let $S(v, w)$ be the surface-area of the best open cell. Since for any fixed value of $v$, $S(v, w)$ is large for both large and small values of $w$, there must be an "absolute best" cell giving the solution of the

Second isoperimetric problem for honeycombs. Among the open cells of volume $v$ generating a honeycomb (of any width) find that one of least surface-area. 
Is the solution of this problem a bee-cell? The answer is: No.

Two bee-cells can be put together with their openings so as to form a "telescopically elongated" rhombic dodecahedron. It is easy to show that among these solids the rhombic dodecahedron (Figure 1a) is the best from the point of view of the isoperimetric problem, i.e., it yields the minimum of the quotient $S^{3} / V^{2}$, where $S$ and $V$ are the surface-area and the volume of the body. The rhombic dodecahedron represents one type of the so-called parallelohedra, or Fedorovean space-fillers, defined as convex polyhedra whose translated replicas can be put together along whole faces so as to fill the space completely. But we know a better parallelohedron than the rhombic dodecahedron, namely the truncated octahedron. Halving the truncated octahedron by a plane orthogonal to one of its hexagonal zones of faces we obtain a cell generating a honeycomb and it may be conjectured that this cell is the solution of our second problem. Anyway the solution is not a half of a rhombic dodecahedron which is the best cell the bees produce while building their cells. The bees start with the bottom and proceed to build the side walls. But they do not stop at the optimal height, when each cell is the half of a rhombic dodecahedron, but continue building rather deep cells. We suppose that they have good reason to do so. Therefore we return to our first problem in the case of large values of $w^{3} / v$.

Also the side walls of a half of a truncated octahedron can be elongated. But having irregular openings these cells cannot compete with those obtained from a rhombic dodecahedron. Yet we can make a trial with a parallelohedron of the type of a truncated octahedron having a regular hexagonal zone of faces.

We elongate the "vertical" diagonal of a regular octahedron symmetrically in both directions so as to obtain an octahedron whose dihedral angles at the horizontal edges are equal to $120^{\circ}$. First we truncate this octahedron by two horizontal planes touching the insphere of the body. The obtained polyhedron is the intersection of two right regular hexagonal prisms. We continue to cut off the remaining corners of the octahedron by planes perpendicular to the corresponding diagonals of the octahedron. Performing this second kind of truncations at a suitable equal depth the hexagonal faces will be centro-symmetric. Since the remaining faces are squares and rhombi all faces will have central symmetry (Figure 2a). Therefore this irregular truncated octahedron is a parallelohedron. It has two squares each of side length, say, $s$. The eight faces adjacent to the squares are hexagons having two opposite sides of length $s$ at a distance $s$ from one another. The diagonal of a hexagon parallel to these sides 


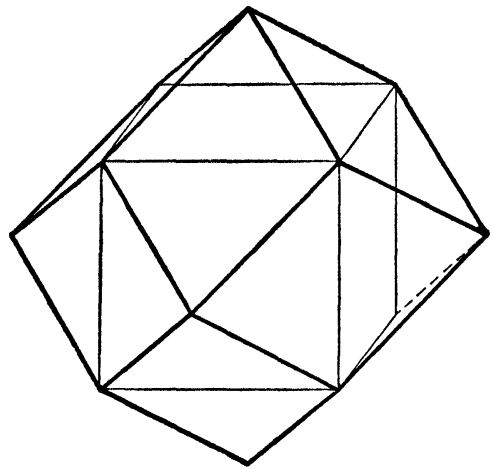

FIGURE 1a

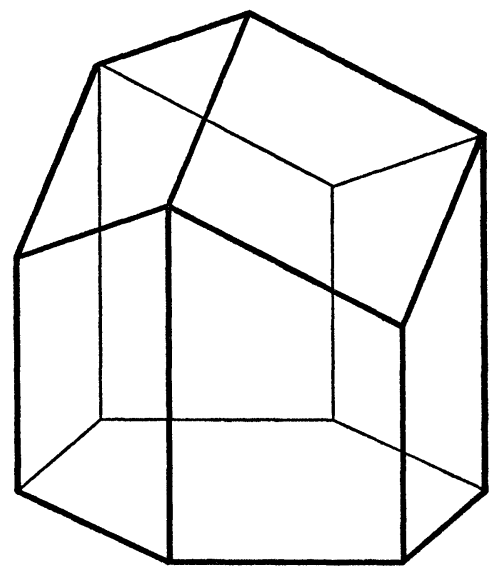

Figure 1b

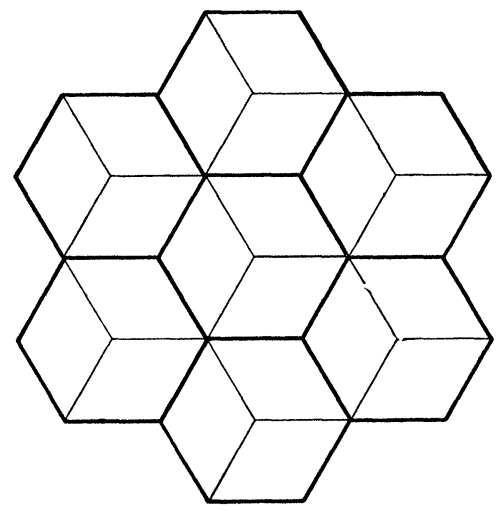

FIGURE 1C

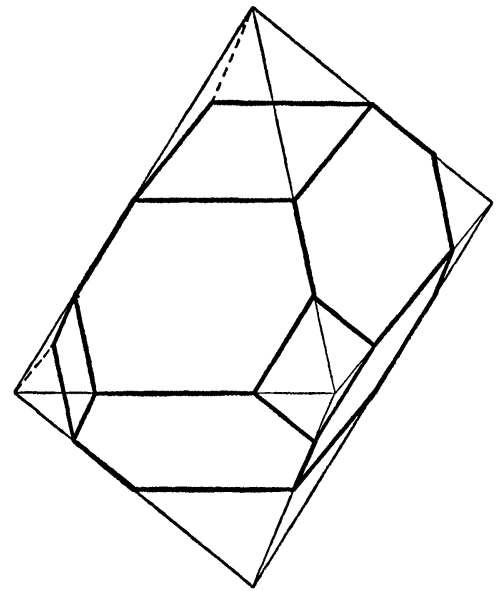

FIGURE 2a

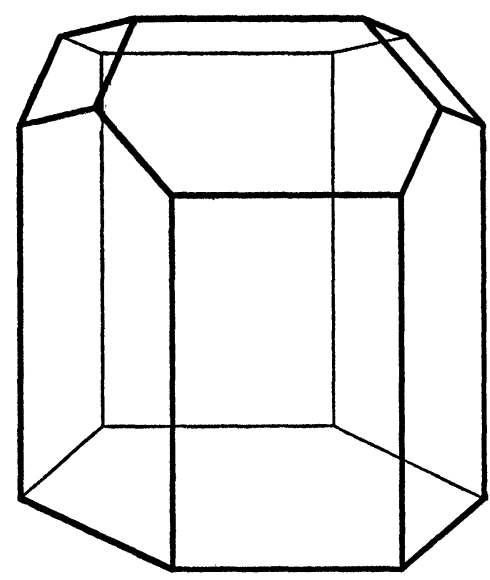

FIGURE 2b

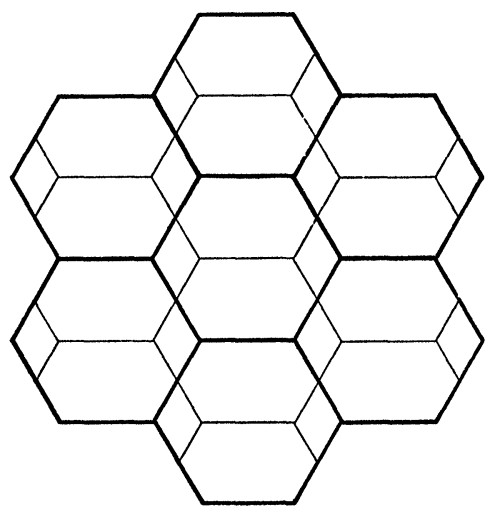

FIGURE 2c 
has a length equal to $3 s / 2$, as can be seen by looking at the polyhedron from the direction of a regular hexagonal zone (Figure 2c). Thus the length of the shorter edges equals

$$
\sqrt{ }\left(\left(\frac{s}{2}\right)^{2}+\left(\frac{s}{4}\right)^{2}\right)=\frac{\sqrt{ }(5) s}{4} .
$$

The polyhedron has four rhombi of side-length $\sqrt{ }(5) s / 4$ and a diagonal of length $\sqrt{ }(3) s / 2$. Thus the length of the other diagonal is $\sqrt{ }(2) s / 2$.

Now we can evaluate the surface-area of our polyhedron:

$$
S=2 s^{2}+8 \frac{5 s^{2}}{4}+4 \frac{\sqrt{ }(6) s^{2}}{8}=\frac{24+\sqrt{ } 6}{2} s^{2} .
$$

The volume $V$ of the polyhedron equals the volume of a regular hexagonal prism having an altitude equal to $3 s / 2$ and edges of length $s$ at the hexagonal faces. Thus $V=9 \sqrt{ }(3) s^{3} / 4$. (It is interesting to note that in the case of unit volume the surface-area

$$
\sqrt[3]{\frac{2}{9}} \frac{24+\sqrt{ } 6}{3}=5.340 \cdots
$$

of our body is less than the surface-area $\sqrt[3]{(108 \sqrt{ } 2)=5.345 \cdots \text { of }}$ the rhombic dodecahedron but greater than the surface-area $\frac{3}{4} \sqrt[3]{4}(1+\sqrt{ } 12)=5.315 \cdots$ of the Archimedean truncated octahedron.)

Letting $s=1$ and elongating our body in the axial direction of one of its regular zones by $x$ we obtain a body of surface-area

$$
S_{x}=\frac{24+\sqrt{ } 6}{2}+6 x
$$

and volume

$$
V_{x}=\frac{9 \sqrt{ } 3}{4}+\frac{3 \sqrt{ } 3}{2} x .
$$

We choose $x$ so that $V_{x}$ equals the volume of a rhombic dodecahedron having a regular hexagonal section of side-length 1 :

$$
\frac{9 \sqrt{ } 3}{4}+\frac{3 \sqrt{ } 3}{2} x=3 \sqrt{ } \frac{3}{2} .
$$

Hence 


$$
x=x_{0}=-\frac{3-\sqrt{ } 8}{2}
$$

showing that the "elongation" is negative. The condition $x>-1$ of performing such a transformation being satisfied we obtain a body of surface-area

$$
S_{x_{0}}=3+\frac{12+\sqrt{ } 3}{\sqrt{ } 2}=12.71002 \ldots
$$

which is less than the surface-area $9 \sqrt{ } 2=12.7279 \cdots$ of the rhombic dodecahedron.

The two kinds of cells which arise by bisecting the rhombic dodecahedron and our shortened snub octahedron have equal volumes and congruent openings. Thus they generate honeycombs of equal width. But the latter has a smaller surface-area than the first and, obviously, the same holds for any elongations of them. As to negative elongations observe that the first cell (arising from the rhombic dodecahedron) can be shortened at most by $\sqrt{ } 2 / 4=0.35 \cdots$ while in the case of the second cell (arising from the shortened snub octahedron) we have a wider latitude for shortening it by $1 / 2-(3-\sqrt{ } 8) / 2$ $=0.41 \cdots$. Thus, recapitulating our result, we can say: Instead of closing the bottom of a cell by three rhombi, as the bees do, it is always more efficient to use two hexagons and two rhombi.

We must admit that all this has no practical consequence. By building such cells the bees would save per cell less than $0.35 \%$ of the area of an opening (and a much smaller percentage of the surfacearea of a cell). On the other hand, the walls of the bee-cells have a non-negligible width which is, in addition, far from being uniform and even the openings of the bee-cells are far from being exactly regular. Under such conditions the above "saving" is quite illusory. Besides, the building style of the bees is definitely simpler than that described above. So we would fail in shaking someone's conviction that the bees have a deep geometrical intuition.

II. Two-dimensional honeycombs, tessellations, drying mud, wallsystems, cellular tissues, foam. The definition of a honeycomb can be extended to any dimensions. In a joint paper Bleicher and I [2] have given a complete enumeration of two-dimensional honeycombs. The types of such honeycombs turned out to be rather limited, so that it was not difficult to pick out the solutions of the isoperimetric problems. Depending on the width of the honeycomb and the area of a cell, the best cells are a half of an elongated regular hexagon or an 

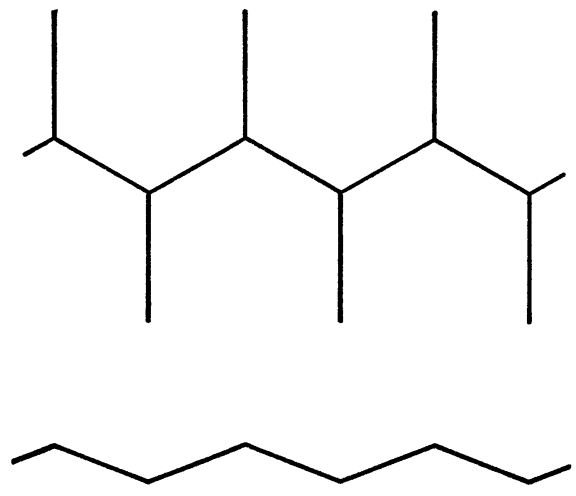

Figure 3
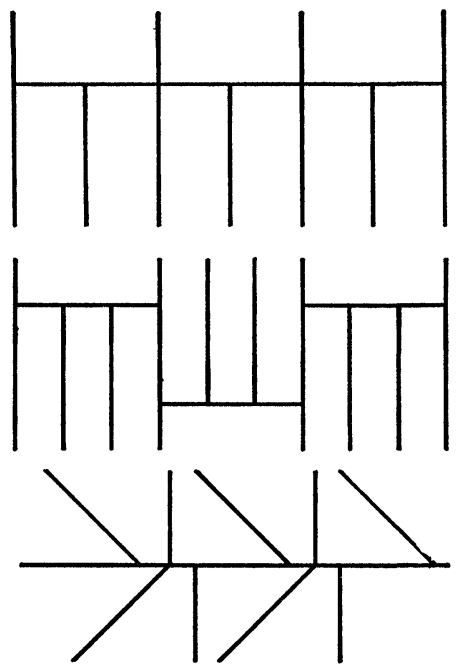

FIGURE 4

isosceles triangle (Figure 3 ). The absolute best cell is, of course, a half of a regular hexagon.

Let us observe that by dropping the second postulate defining a honeycomb we obtain "unnatural" honeycombs like those exhibited in Figure 4. However, this does not change the solutions of the isoperimetric problems.

What happens if we even drop the congruence of the cells and consider only cells of equal and given area? We can then inquire about the minimum of the average perimeter of the cells defined by a suitable limiting value. For incongruent cells a second problem arises which is the dual counterpart of the first one. We can consider cells of equal and given perimeter and ask about the maximum of the average area of the cells. It would be nice to prove that the solutions are, in both problems, the same as for congruent cells (in which case the two problems are equivalent).

The analogous problems for tessellations are as follows.

(a) Decompose the Euclidean plane into parts of unit area so that the average perimeter of the parts should be as small as possible.

(p) Decompose the Euclidean plane into parts of unit perimeter so that the average area of the parts should be as great as possible.

It is known [3] that under the restriction to convex cells the solution of problem (a) is the regular hexagonal tessellation. There is no doubt that the same is true for general cells. Nevertheless, this conjecture resisted all attempts at proving it. On the other hand, prob- 
lem (p) is solved in its full generality [4]. The solution is again the regular hexagonal tessellation.

These extremal properties of the hexagonal tessellation may be extended to its spherical and hyperbolic analogues, namely to the regular tessellations with trihedral vertices. As to problem (a) we mention the following theorem [5].

If $U$ is the union of $n$ cells of a regular (spherical, Euclidean or hyperbolic) tessellation with trihedral vertices then among the decompositions of $U$ into $n$ convex cells of equal area the regular decomposition has the least total edge-length.

The difficulties involved in the problem of generalizing this theorem to nonconvex cells is illustrated by the following interesting remark due to A. Heppes: If $n$ is a positive integer different from 2, 3, 4, 6 and 12 then the shortest net which decomposes the sphere into $n$ parts of equal area necessarily contains a nonconvex mesh.

What is the situation if, e.g., $\dot{n}=12$ ? Are all meshes convex? If so then, in view of the above theorem, they must be regular pentagons.

The following theorem [4] involves the solution of problem (p).

Let $U$ be the union of $n$ faces of a regular Euclidean or hyperbolic tessellation with trihedral vertices. If we decompose $U$ in any way into $n$ connected parts the perimeter of the part of greatest perimeter attains its minimum for the regular decomposition.

For spherical tessellations this theorem must be modified. If, namely, $U$ consists of the whole sphere no part is allowed to have a greater area than a hemisphere.

In his book Kaleidoskop der Mathematik (Berlin, 1959), H. Steinhaus gives a "theory" of the breaking lines arising on a drying table of mud. Similar lines may be seen on potteries covered with special glaze which contracts considerably when drying. We have some reason to suppose that these lines come into being successively so that at each turn one of the parts of greatest area splits along a shortest line into two parts of equal area. What can be said about the total length of the lines after the $n$th breaking?

Let $P_{n}$ be the sum of the perimeters of the parts after a decomposition into $n$ parts. It is easy to show that if the original domain is a rectangle of area $A$, we have

$$
\begin{aligned}
4 \sqrt{ } A & \leqq \liminf _{n \rightarrow \infty} \frac{P_{n}}{\sqrt{ } n} \leqq 2\left(2^{1 / 4}+2^{-1 / 4}\right) \sqrt{ } A, \\
2\left(2^{1 / 4}+2^{-1 / 4}\right) \sqrt{ } A & \leqq \limsup _{n \rightarrow \infty} \frac{P_{n}}{\sqrt{ } n} \leqq \sqrt{ } 18 \sqrt{ } A .
\end{aligned}
$$


The bound $4 \sqrt{ } A$ corresponds to the case when the partial domains are squares. Of course, even in this best situation of a successive decomposition the total perimeter is greater than it is in an immediate decomposition into hexagons.

Do these inequalities hold for any domain of area $A$ ? Probably, yes. This may be conjectured from the fact that, starting with any domain, for great values of $n$ the bulk of the partial domains will have a nearly rectangular shape.

After this digression we return to problem (a) whose connection with an economical building of a honeycomb consisting of incongruent cells is obvious. We restrict ourselves to convex cells; on the other hand, we want to take into consideration the width of the cellwalls.

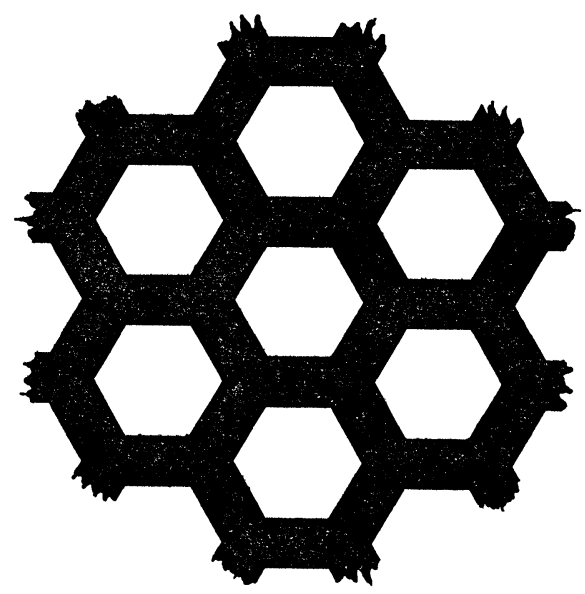

Figure 5

We define a wallwork as a connected domain bounded by at least two closed curves. Among these curves there is one, the contour of the wallwork, which encloses the whole wallwork. The domains enclosed by the remaining curves are called cells. The width of the wallwork is the minimal distance between two points belonging to different bounding curves.

Now we want to construct a wallwork of given contour and given width containing as great a number of convex cells of given area as possible. This amounts to making the area of the wallwork small. The following theorem [6] shows that for a "big" contour the solution is a regular hexagonal wallwork (Figure 5).

Consider a wallwork of width $2 t$ lying in a convex hexagon of area $H$. If the wallwork has $n$ convex cells all of area $\geqq h$, then 


$$
n \leqq\left(\frac{\sqrt{ } H-\sqrt[4]{ }(12) t}{\sqrt{ } h+\sqrt[4]{ }(12) t}\right)^{2}
$$

Here a hexagon means a polygon with at most six sides. Note that $(\sqrt{ } A \pm \sqrt[4]{ }(12) t)^{2}$ equals the area of a regular hexagon arising from another one of area $A$ by displacing each side by $t$ outward or inward, respectively.

In certain cellular tissues the cells do not completely fill the available space. The consideration of such tissues leads to new mathematical problems. We consider the tissue as consisting of nonoverlapping plastic cells all contained in a given part of space. Supposing first that the cells have the same constant volume, we can ask what shape and arrangement the cells must assume in order to make their total surface-area as small as possible. Supposing, secondly, that the cells have equal and unchanged surface-area, we can ask in what shape and arrangement the total volume of the cells will be as great as possible.

In the stem of certain plants the cells may be considered as small columns considerably elongated in the axial direction so that their volume and surface-area may be supposed to be proportional to the area and perimeter of their sections. This suggests the following twodimensional analogues of the above problems.

(A) Consider in the Euclidean plane a set of nonoverlapping discs of unit area having a given number-density. Find the shape and the arrangement of the discs in which their average perimeter attains its minimum.

(P) Consider in the Euclidean plane a set of nonoverlapping discs of unit perimeter having a given number-density. Find the shape and the arrangement of the discs in which their average area attains its maximum.

The number-density of a set of discs scattered over the plane is defined by a suitable limiting value and may be interpreted as the number of discs per unit of area. Thus, instead of considering an infinite set of discs of given number-density, we can, roughly speaking, consider a great number of discs lying in a given circle (or in any domain of fixed shape). Obviously, the problems (A) and (P) are generalizations of the problems (a) and (p).

In the case of convex discs problem $(\mathrm{P})$ was solved in a paper of the author [7] and problem (A) in a common paper of the author and Heppes [9]. For small values of the number-density $d$ the extremal discs are circles arranged in an arbitrary way. Increasing $d$, the circles will get into hexagonal close-packing and we know that, in some 


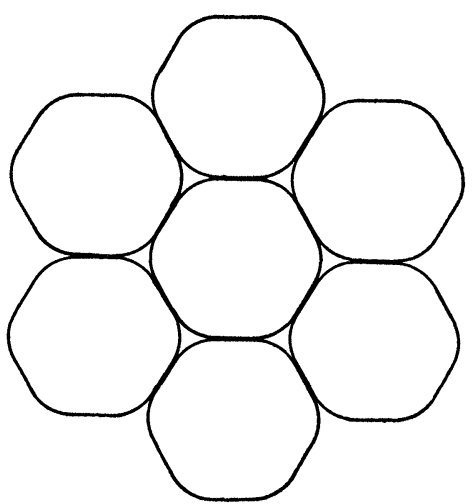

Figure 6

way, they must turn into regular hexagons. How does this transition go? The answer is the same in both problems: through smooth hexagons, each arising from a regular hexagon by rounding off its corners by equal circular arcs (Figure 6).

Recently, Heppes [10] pointed out that in the case of problem (P) we obtain the same solution without restricting ourselves to convex discs. But in the general case of problem (A) we encounter the same difficulties arising in problem (a).

For convex domains the results mentioned in connection with the problems (a) and ( $p$ ) may be generalized in another direction. We consider a decomposition of the Euclidean plane into convex cells. Let $a$ be the area and $p$ the perimeter of a cell. Our results [8], [4] concern the mean-values $\overline{p / \sqrt{ } a}$ and $\overline{a / p^{2}}$ of the quotients $p / \sqrt{ } a$ and $a / p^{2}$ extended over all cells. If these mean-values exist, then

$$
\overline{p / \sqrt{ } a} \geqq 2 \sqrt[4]{12}
$$

and

$$
\overline{a / p^{2}} \leqq 1 / 8 \sqrt{ } 3 .
$$

If for each cell $a=1$ or $p=1$, then $\overline{2} \geqq 2 \sqrt{ } 12$ and $a \leqq 1 / 8 \sqrt{ } 3$, respectively, in accordance with the results mentioned above.

The inequalities (a) and ( $p$ ) are valid also for a decomposition of any convex hexagon into convex pieces. Since the quadratic mean is larger than the arithmetic mean (a) gives a lower bound for the average isoperimetric quotient of the partial domains:

$$
\overline{p^{2} / a} \geqq 8 \sqrt{ } 3 \text {. }
$$


This weaker inequality may be generalized in another direction [8]: it holds with strict inequality for the decomposition of any convex domain into at least two convex partial domains. To refer to related fields, we mention an immediate consequence of this result according to which a convex domain can never be packed as densely by equal circles as the whole plane.

We have considered the total length of certain spherical nets. An interesting ramification of this problem concerns the total edgelength of a polyhedron containing a given sphere. The discussion of the various results obtained in this direction and the problems to be solved would lead too far afield. Omitting this we turn our attention to solid tessellations.

Besides the volume $V$ and the surface-area $S$, a convex polyhedron has a third fundamental gauge, the edge-curvature $M$ defined by

$$
M=\frac{1}{2} \sum \alpha l,
$$

where $l$ is the length of an edge, $\alpha$ the outer dihedral angle at this edge, and the summation extends over all edges. Therefore the isoperimetric problems which may be raised for tessellations are much more varied in space than in the plane. In the problems concerning solid tessellations there is also a fundamental difference between convex and general cells. This difference concerns not only the diffculty of the solutions but the solutions themselves. We start by considering convex cells.

In the "immediate" analogues of the two-dimensional problems, involving $V$ and $S$ only, the part of the regular hexagon seems to be taken over by the Archimedean truncated octahedron. But this is not always so. The following inequality [8] settles a problem the solution of which is dominated by the architecture of the bees.

Consider a decomposition of the Euclidean space into convex cells. Let $V, S$ and $M$ be the volume, surface-area and edge-curvature of a cell and suppose that the mean-values $\overline{S^{2} / V}$ and $\bar{M}$ exist. Then

$$
\pi \overline{S^{2} / V} \geqq 6 \sqrt{ } 3 \bar{M} .
$$

We emphasize the special case of congruent cells: The three fundamental gauges of a convex space-filler satisfy the inequality

$$
\pi S^{2} \geqq 6 \sqrt{ } 3 M V .
$$

Equality holds for exactly two bodies, namely for the rhombic dodecahedron and the trapezo-rhombic dodecahedron (which arises from the rhombic dodecahedron by cutting it by a plane perpendicular to an edge into two equal parts and replacing one part by the 
image of the other part reflected in this plane). These two solids may be defined as polyhedra circumscribed about a sphere all dihedral angles of which are $120^{\circ}$.

We recall the classical problem of finding the complete system of inequalities which hold between the fundamental gauges of a convex body. This exciting but extremely difficult problem, brought into prominence by Blaschke, is not solved completely as yet. The above inequality suggests the more hopeful problem for the Fedorovean (or perhaps for all) space-fillers.

Now we consider the problem of decomposing the space into not necessarily convex cells of unit volume having the least average surface-area. It can be shown that in a best decomposition along each edge three faces must meet under equal angles. To give this statement a more detailed formulation, note that from a topological point of view the cells are polyhedra with generally curved faces and edges. Thus along the edges the dihedral angle may vary from point to point. But in the extremal case the dihedral angles have the constant value of $120^{\circ}$.

Though the dihedral angles of the rhombic dodecahedron are equal to $120^{\circ}$, we know that the decomposition into truncated octahedra is better. On the other hand, the dihedral angles of the truncated octahedron are not equal to $120^{\circ}$. Therefore this decomposition is not the best one either. In fact, Lord Kelvin showed that the truncated octahedron can be deformed into a nonconvex space-filler of the same volume but smaller surface-area. Has Lord Kelvin's solid the least surface-area among the space-fillers of equal volume? Or is the solid tessellation generated by it even the solution of the general problem involving incongruent cells? Our present mathematical knowledge is inadequate to answer these questions.

To conclude, we mention a highly interesting result due to Heppes which may be interpreted as follows. If in a lather there is a bubble surrounded entirely by other ones then the lather contains a nonconvex bubble. The exact statement reads as follows. Let $U$ be the union of $n$ convex bodies of volume $V_{1}, \cdots, V_{n}$. If there is a body lying completely in the interior of $U$, then $U$ can be decomposed into $n$ partial bodies of volume $V_{1}, \cdots, V_{n}$ having a smaller total surfacearea than the original bodies. (To make the above interpretation clear we must add that such new bodies may be obtained by arbitrarily small variations of the original ones.)

The ingenious proof rests on the enumeration of all spherical tessellations with convex faces each angle of which is equal to $2 \pi / 3$. It turned out that besides the four regular tessellations of this type there 
are five irregular ones. Since the further possibilities when all angles are $2 \pi / 4,2 \pi / 5, \cdots$ yield only regular tessellations, Heppes obtained, as an additional result, the enumeration of all isogonal spherical tessellations with convex faces.

Of course, the theorem of Heppes does not hold in spherical or hyperbolic spaces. Consider, for instance, the famous decomposition of the spherical space into 120 regular dodecahedra. There is no doubt that of all decompositions into 120 parts of equal volume the regular decomposition has the least total surface-area of the parts. Unfortunately, the proof of this conjecture involves considerable difficulties.

\section{BIBLIOGRAPHY}

1. W. D'Arcy Thompson, On growth and form. I, II, 2nd ed., Cambridge Univ. Press, Cambridge, 1952.

2. M. N. Bleicher and L. Fejes Tóth, Tro-dimensional honeycombs, Amer. Math. Monthly (to appear).

3. L. Fejes T6th, Über das kürzeste Kurvennetz, das eine Kugeloberfläche in flächengleiche konvexe Teile zerlegt, Mat. Term.-tud. Értesitö 62 (1943), 349-354.

4. - Isoperimetric problems concerning tessellations, Acta Math. Acad. Sci. Hungar. 14 (1963), 343-351.

5. - On shortest nets with meshes of equal area, Acta Math. Acad. Sci. Hungar. 11 (1960), 363-370.

6. - An arrangement of two-dimensional cells, Ann. Univ. Sci. Budapest. Eötvös Sect. Math. 2 (1959), 61-64.

7. - Filling of a domain by isoperimetric discs, Publ. Math. Debrecen 5 (1957), 119-127.

8. - - Lagerungen in der Ebene, auf der Kugel und im Raum, Springer, Berlin, 1953.

9. L. Fejes Tóth and A. Heppes, Filling of a domain by equiareal discs, Publ. Math. Debrecen 7 (1960), 198-203.

10. A. Heppes, Filling of a domain by discs, Publ. Math. Decebren (to appear).

UNIVERSITY OF WisCONSIN 